\title{
OPERATOR THEORETICAL REALIZATION OF SOME GEOMETRIC NOTIONS
}

\author{
LIN QING
}

\begin{abstract}
This paper studies the realization of certain geometric constructions in Cowen-Douglas operator class. Through this realization, some operator theoretical phenomena are easily seen from the corresponding geometric phenomena. In particular, we use this technique to solve the first-order equivalence problem and introduce a new operation among certain operators.
\end{abstract}

The nature of Cowen-Douglas theory is to identify operators of a certain type with certain geometric objects.

Based on this idea, we work on certain geometric constructions, holomorphic curves in $\operatorname{Gr}\left(n, \mathbf{C}^{2 n}\right.$ ) (the Grassmannian of $n$-dim subspaces of $\mathbf{C}^{2 n}$ ) in Part 1 and tensor product of vector bundles in Part 2, and seek their operator theoretical realization.

Our realization of holomorphic curves in $\operatorname{Gr}\left(n, \mathbf{C}^{2 n}\right)$ will preserve important relations, and can be informally viewed as the imbedding of holomorphic curves in $\operatorname{Gr}\left(n, \mathbf{C}^{2 n}\right)$ into the Cowen-Douglas operator class $B_{n}(\Omega)$. Using this realization, we solve the first-order equivalence problem by explicitly exhibiting two operators $T_{1}, T_{2} \in B_{n}(D)$ such that $T_{1}$ is not unitarily equivalent to $T_{2}$ but $T_{1}$ and $T_{2}$ have identical curvatures.

The realization of tensor product of vector bundles gives a natural operation among Cowen-Douglas operators. Using this operation, certain operator theoretical phenomena have been clarified naturally. E.g., for certain $g \in H^{\infty}$, the corresponding Bergman operator $B_{g}^{*}$ is the "square" of the corresponding Toeplitz operator $T_{g}^{*}$.

This paper is part of the author's Ph.D. dissertation. I dedicate this paper to my thesis advisor Michael J. Cowen.

Also, I would like to thank L. A. Coburn, R. E. Curto and W. L. Paschke for the various help I received during the preparation of this paper.

\section{PART 1. OPERATOR THEORETICAL REALIZATION OF HOLOMORPHIC CURVES IN $\operatorname{Gr}\left(n, \mathbf{C}^{2 n}\right)$}

1.1. Introduction. We will state only the main point of Cowen-Douglas theory here, and refer the reader to $[\mathbf{C}-\mathbf{D}, \mathbf{1}]$ for further details.

If $H$ is a separable Hilbert space, and $\Omega$ is an open connected subset of $\mathbf{C}$, then the operator class $B_{n}(\Omega)$ is by definition

$$
\begin{array}{ll}
\{T \in \mathcal{L}(H): & \text { 1. } \quad \operatorname{range}(T-w)=H, \text { if } w \in \Omega \\
& \text { 2. } \quad \operatorname{dim} \operatorname{ker}(T-w)=n, \text { if } w \in \Omega ; \\
& \text { 3. } \left.\bigvee_{w \in \Omega} \operatorname{ker}(T-w)=H \text { (spamning property) }\right\} .
\end{array}
$$

Received by the editors November 18, 1986.

1980 Mathematics Subject Classification (1985 Revision). Primary 47B99; Secondary 47B35. 
We call the operators in $B_{n}(\Omega)$ Cowen-Douglas operators.

The fundamental relation between $T \in B_{n}(\Omega)$ and the associated $n$-dim holomorphic Hermitian vector bundle over $\Omega$ defined by

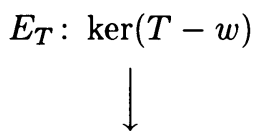

$w$

is the following identification:

THEOREM A $[\mathbf{C - D}, \mathbf{1}]$. Two operators $T$ and $\tilde{T}$ in $B_{n}(\Omega)$ are unitarily equivalent $\Leftrightarrow E_{T}$ and $E_{\tilde{T}}$ are equivalent as holomorphic Hermitian vector bundles. We write $E_{T} \cong E_{\tilde{T}}$.

On the other hand, the Calabi Rigidity Theorem gives a perfect identification of a holomorphic curve (with spanning property) ${ }^{1} \gamma: \Omega \rightarrow \operatorname{Gr}\left(n, \mathbf{C}^{2 n}\right)$ with its pull-back of the universal subbundle (i.e. $\gamma^{*}\left(S\left(n, \mathbf{C}^{2 n}\right)\right)$ ).

Our philosophy is they are related through their identified vector bundles.

DEFINITION 1.1.1. Let $F=\left(f_{i j}\right)_{i, j}$ be a $n \times n$ matrix with $H^{\infty}$ entries and let $g \in H^{\infty}$; we define the operator $S(g, F)$ by

$$
\left.S(g, F) \stackrel{\text { def }}{=}\left(T_{g \otimes I_{n}}^{*} \oplus T_{g \otimes I_{n}}^{*}\right)\right|_{\operatorname{Graph}\left(T_{F}^{*}\right)}
$$

where $T_{F}^{*}$ and $T_{g \otimes I_{n}}^{*}$ are matrix Toeplitz operators acting on row vectors in $H^{2} \otimes \mathbf{C}^{n}$.

It turns out that $S(g, F)$ is the right operator realization of the holomorphic curve, $\operatorname{span}\left({ }_{F}^{I}\right): D \rightarrow \operatorname{Gr}\left(n, \mathbf{C}^{2 n}\right)$, where $D$ is the open unit disk.

The geometric nature of $S(g, F)$ will be discussed in $\S 1.2$ and its operator theoretical nature will be discussed in $\S 1.4$.

(In this paper, the geometric part of a Cowen-Douglas operator or of a holomorphic curve means the geometric part of its corresponding vector bundle.)

1.2. Geometric aspects of this realization. In this section, we will show that for certain $g \in H^{\infty}$, the associated operator $S(g, F)$ belongs to $B_{n}(\Omega)$, and that through this realization, i.e. from the pull-back of the universal subbundle by $\operatorname{span}\left({ }_{F}^{I}\right): D \rightarrow \operatorname{Gr}\left(n, \mathbf{C}^{2 n}\right)$ to $E_{S(g, F)}$, the important geometric relations are preserved.

In order to do this, we need to recall the definitions of some geometric invariants.

Let $E, \tilde{E}$ denote two $n$-dim Hermitian holomorphic vector bundles over an open connected set $\Omega \subset \mathrm{C}$. Let $D_{E}$ denote the canonical connection of $E$ and $D_{E}^{2}=K_{E} d z d \bar{z}$ be its curvature tensor. We sometimes write $K_{E}$ as $K$ when no confusion arises.

It is well known that $K$ is a $C^{\infty}$ selfadjoint bundle map of $E$ to $E$.

DEFINITION 1.2.1. If $\phi: E \rightarrow E$ is a $C^{\infty}$ bundle map, then define $\phi_{z}$ and $\phi_{\bar{z}}$ by

$$
\left[D_{E}, \phi\right]=D_{E} \phi-\phi D_{E}=\phi_{z} d z+\phi_{\bar{z}} d \bar{z}
$$

$\left[D_{E}, \phi\right]$ is a $C^{\infty}$ bundle map of $E$ to $E \otimes \mathcal{E}^{\prime}(\Omega)$, where $\mathcal{E}^{\prime}(\Omega)$ denotes the set of $C^{\infty}$ 1 -forms over $\Omega$.

\footnotetext{
${ }^{1} \mathrm{~A}$ holomorphic curve $\gamma$ in $\operatorname{Gr}\left(n, \mathbf{C}^{2 n}\right)$ has the spanning property if $\sum_{z \in \Omega} \gamma(z)=\mathbf{C}^{2 n}$. In this paper, we only consider holomorphic curves with this propety.
} 
Here $\phi_{z}, \phi_{\bar{z}}$ are clearly bundle maps of $E \rightarrow E$; they are called the first covariant derivatives for $\phi$.

Taking the first covariant derivatives of $\phi_{z}, \phi_{\bar{z}}$ and taking covariant derivatives of their covariant derivatives, etc., we get higher order covariant derivatives of $\phi$.

For details see [C-D, 2].

REMARK. Relative to any $C^{\infty}$ frame $S$ of $E$, write $\Gamma(S) d z+\tilde{\Gamma}(S) d \bar{z}$ for the connection 1-form matrix of $D_{E}$ and $\phi(S)$ for the matrix representation of $\phi$ relative to $S$. Then

$$
\phi_{\boldsymbol{z}}(S)=[\Gamma(S), \phi(S)]+\partial \phi(S) / \partial z, \quad \phi_{\bar{z}}(S)=[\tilde{\Gamma}(S), \phi(S)]+\partial \phi(S) / \partial \bar{z} .
$$

The covariant derivatives of the curvature bundle map $K_{E}$ give the important geometric invariants of $E$.

DEFINITION 1.2.2. Let $E, \tilde{E}$ be $n$-dim Hermitian holomorphic vector bundles and let $k$ be a positive integer. We say $E$ is equivalent to order $k$ with $\tilde{E}$, if for each $z \in \Omega$, there is an isometry $\phi_{z}: E_{z} \rightarrow \tilde{E}_{z}$ such that $\phi_{z} \circ \chi=\tilde{\chi} \circ \phi_{z}$, where $\chi$ is a covariant derivative of $K$ with total order $\leq k$, but bi-order $(p, q) \neq(0, k)$ or $(k, 0)$, and $\tilde{\chi}$ is the corresponding covariant derivative for $\tilde{K}$. (We shall say $\chi$ has total order $\leq k$ and satisfies the bi-order condition.)

For example, $E$ is equivalent to order 1 with $\tilde{E} \Leftrightarrow$ for each $z \in \Omega$, there is an isometry $\phi_{z}: E_{z} \rightarrow \tilde{E}_{z}$ such that $\phi_{z} \circ K=\tilde{K} \circ \phi_{z}$. (We say $E$ and $\tilde{E}$ have identical curvatures.)

TheOREM B $[$ C-D, 2]. If $\operatorname{dim} E=\operatorname{dim} \tilde{E}=n$, then $E \cong \tilde{E} \Leftrightarrow E$ and $\tilde{E}$ are equivalent to order $n$.

We list two simple facts related to Definition 1.2.2:

(1) If $\tilde{\Omega}, \Omega \subset \mathbf{C}, g: \tilde{\Omega} \rightarrow \Omega$ is an analytic function, then $E_{1}$ and $E_{2}$ are equivalent to order $k$, so are $g^{*}\left(E_{1}\right)$ and $g^{*}\left(E_{2}\right)$.

(2) If $E_{1}$ and $\tilde{E}_{1}, E_{2}$ and $\tilde{E}_{2}$ are both equivalent to order $k$ respectively, so are $E_{1} \otimes E_{2}$ and $\tilde{E}_{1} \otimes \tilde{E}_{2}$.

For an explanation of this, see $[\mathbf{L}]$.

If $T_{1}$ and $T_{2}$ are in $B_{n}(\Omega)$, the relation of $E_{T_{1}}$ and $E_{T_{2}}$ being equivalent to order $k$ is directly reflected in the relation of $T_{1}$ and $T_{2}$.

THEOREM C $[\mathbf{C - D}, \mathbf{1}]$. If $T_{1}, T_{2} \in B_{n}(\Omega)$, then $E_{T_{1}}$ and $E_{T_{2}}$ are equivalent to order $\left.k \Leftrightarrow T_{1}\right|_{\operatorname{ker}\left(T_{1}-w\right)^{k+1}}$ and $\left.T_{2}\right|_{\operatorname{ker}\left(T_{2}-w\right)^{k+1}}$ are unitarily equivalent for each $w \in \Omega$.

In this situation, we will say $T_{1}$ and $T_{2}$ are equivalent to order $k$.

Notation. We will use $\bar{\Omega}$ to denote the conjugate of a subset $\Omega$ of $\mathbf{C}$ and $\operatorname{bd}(D)$ to denote the boundary of $D$.

The following lemma is a characterization of $T_{g}^{*} \in B_{1}(\Omega)$ for $g \in H^{\infty}$.

LEMMA 1.2.1. If $g \in H^{\infty}, \Omega$ connected open in $\mathbf{C}$, then $T_{g}^{*} \in B_{1}(\Omega) \Leftrightarrow$ the map $g: g^{-1}(\bar{\Omega}) \rightarrow \bar{\Omega}$ is onto and is a conformal equivalence.

ProOF. Recall

$$
\left(T_{g}^{*}-\overline{g(\bar{z})}\right) k_{z}=0,
$$

where $z \in D$ and $k_{z}(\zeta)=1 /(1-z \zeta)$ for $\varsigma \in D$. 
" $\Rightarrow$ " The mapping $g: g^{-1}(\bar{\Omega}) \rightarrow \bar{\Omega}$ has to be injective, because $z_{1} \neq z_{2}$ in $D$ implies $k_{z_{1}}$ and $k_{z_{2}}$ are linearly independent.

The fact that $g=g^{-1}(\bar{\Omega}) \rightarrow \bar{\Omega}$ is surjective follows from:

(1) $\bar{\Omega} \subset \overline{\sigma\left(T_{g}^{*}\right)}=\sigma\left(T_{g}\right)=\operatorname{clos}(g(D))$;

(2) $\bar{\Omega} \cap \sigma_{e}\left(T_{g}\right)=\overline{\Omega \cap \sigma_{e}\left(T_{g}^{*}\right)}$ is empty;

(3) $\sigma_{e}\left(T_{g}\right) \supset \operatorname{bd}(g(D))$. (See [D].)

" $\Leftarrow "$ Step 1 . Fix any $w=g\left(z_{0}\right) \in \bar{\Omega}$, then $g(z)-w=\left(z-z_{0}\right) h(z)$.

We claim $h$ is invertible in $H^{\infty}$.

It is trivial to see $h \in H^{\infty}$ and $h$ is nowhere zero in $D$.

The invertibility of $h$ in $H^{\infty}$ follows from observing that for any $z_{n} \in D$, with $g\left(z_{n}\right) \rightarrow w$ (assume $g\left(z_{n}\right) \in \bar{\Omega}$ ), we have $g^{-1}\left(g\left(z_{n}\right)\right)=z_{n} \rightarrow z_{0}$ (because $g: g^{-1}(\bar{\Omega}) \rightarrow \bar{\Omega}$ is a conformal equivalence).

Step 2. Let $w=g\left(z_{0}\right) \in \bar{\Omega}$ as above. Notice that $\operatorname{ker}\left(T_{g}-w\right)=0$.

We claim range $\left(T_{g}-w\right)=\left\{f \in H^{2}: f\left(z_{0}\right)=0\right\}$.

This follows from two facts:

(1) $\left[\left(T_{g}-w\right)(f)\right](z)=(g(z)-w) f(z)=\left(z-z_{0}\right) h(z) f(z)$;

(2) if $f(z)=\left(z-z_{0}\right) l(z)$ with $l \in H^{2}$, then

$$
f(z)=\left[\left(z-z_{0}\right) h(z)\right](l(z) / h(z))=\left[\left(T_{g}-w\right)(l / h)\right](z) .
$$

Thus $f-\left(f\left(z_{0}\right) / k_{\bar{z}_{0}}\left(z_{0}\right)\right) k_{\bar{z}_{0}} \in \operatorname{range}\left(T_{g}-w\right)$. Thus $T_{g}-w$ has closed range and using formula $(*)$, we have

$$
\operatorname{span}\left(k_{\bar{z}_{0}}\right) \oplus \operatorname{range}\left(T_{g}-w\right)=H^{2} .
$$

So $\operatorname{dim}\left(\operatorname{ker}\left(T_{g}^{*}-\bar{w}\right)\right)=\operatorname{dim}\left[\operatorname{coker}\left(T_{g}-w\right)\right]=1$, and $T_{g}^{*}-\bar{w}$ is Fredholm of index 1.

This shows $T_{g}^{*} \in B_{1}(\Omega)$.

From now on, $E_{F}$ will denote the holomorphic Hermitian vector bundle

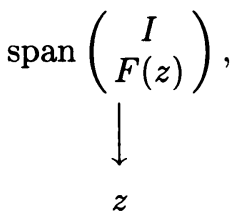

where $F=\left(f_{i j}\right)_{i, j}$ is an $n \times n$ matrix of analytic functions on $\Omega \subset \mathbf{C} ;\left(\begin{array}{c}I \\ F(z)\end{array}\right)$ is always viewed as a collection of column vectors in $\mathbf{C}^{2 n}$.

THEOREM 1.2.2. If $g \in H^{\infty}$ and $T_{g}^{*} \in B_{1}(\Omega)$, then $S(g, F) \in B_{n}(\Omega)$ for any $F=\left\{f_{i j}\right\}_{i, j}$ with each $f_{i j} \in H^{\infty}$. Moreover

1. $E_{S(g, F)} \cong E_{T_{g}^{*}} \otimes E_{\text {子 }}$, where $\mathcal{F}(z)=\overline{F\left(g^{-1}(\bar{z})\right)}$;

2. $E_{F}$ and $E_{G}$ are equivalent to order $k \Leftrightarrow S(g, F)$ and $S(g, G)$ are equivalent to order $k$ (where $G$ has entries in $H^{\infty}$ ).

ProOF. It is trivial to see that $S(g, F) \in B_{n}(\Omega)$, since $B_{n}(\Omega)$ is closed under similarity and $S(g, F) \sim T_{g \otimes I_{n}}^{*}$ (by the graph mapping $x \mapsto\left(x, T_{F}^{*} x\right)$ ). We go directly to 1 and 2 . 
1. Observe that $k_{g^{-1}(\bar{z})}$ is a holomorphic frame of $E_{T_{g}^{*}}\left(k_{w}(\varsigma)=1 /(1-\varsigma w)\right)$,

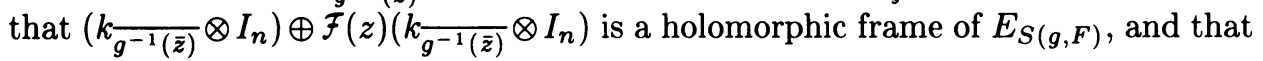
$\left(\begin{array}{c}I \\ (z)\end{array}\right)$ is a holomorphic frame of $E_{\mathcal{F}}$. Therefore

$$
\left(k_{g^{-1}(\bar{z})} \otimes I_{n}\right) \oplus \mathcal{F}(z)\left(k_{\overline{g^{-1}(\bar{z})}} \otimes I_{n}\right) \rightarrow k_{g^{-1}(\bar{z})} \otimes\left(\begin{array}{c}
I \\
\mathcal{F}(z)
\end{array}\right)
$$

is the desired holomorphic isometric bundle map. (In the expression above, both sides are thought of as collections of $n$-vectors.)

2. Using the fact (2) following Definition 1.2.2, we see $E_{\text {₹ }}$ is equivalent to order $k$ with $E_{\mathcal{G}} \Leftrightarrow E_{S(g, F)}$ and $E_{S(g, G)}$ are equivalent to order $k$, where $\mathcal{G}(z)=\overline{G\left(g^{-1}(\bar{z})\right)}$.

Once we prove " $E_{F}$ and $E_{G}$ are equivalent to order $k \Leftrightarrow E_{\xi}$ and $E_{\mathcal{G}}$ are equivalent to order $k$ for $g(z) \equiv z$," then the rest follows directly from fact (1).

NOTE. Over the holomorphic frame $\left(\begin{array}{c}I \\ F(z)\end{array}\right)$, the connection 1-form matrix of $E_{F}$ is $\left\{\left(I+F^{*}(z) F(z)\right)^{-1} F^{*}(z) F^{\prime}(z)\right\} d z$ and so the matrix of its curvature bundle map $K_{E_{F}}$ is

$$
-\left(I+F^{*}(z) F(z)\right)^{-1}\left(F^{\prime}(z)\right)^{*}\left(I+F(z) F^{*}(z)\right)^{-1} F^{\prime}(z) \quad(\text { see }[\mathbf{C}-\mathbf{D}, \mathbf{1}]) .
$$

So by the remark following Definition 1.2.1, over the holomorphic frame $\left(\begin{array}{c}I \\ F(z)\end{array}\right)$ the matrix representations of the covariant derivatives of $K_{E_{F}}$ on $E_{F}$ are all noncommutative polynomials in $F^{(i)}(z), \overline{F^{(j)}(z)}$ and $\left(I+F^{*}(z) F(z)\right)^{-1},\left(I+F(z) F^{*}(z)\right)^{-1}$, $i, j \geq 0$. Also such polynomials are canonical in the sense that they are independent of the choice of $F$. So for a covariant derivative of $E_{F}$, the conjugate of its matrix (relative to the frame $\left(\begin{array}{l}I \\ F\end{array}\right)$ ) at $\bar{z}$ is exactly the corresponding one for $E_{\mathcal{F}}$ (relative to the frame $\left.\left(\begin{array}{l}I \\ \xi\end{array}\right)\right)$ at $z$.

From Definition 1.2.2, the rest of the proof is quite straightforward.

Notice that $E_{F}$ is the pull-back of the universal bundle under the holomorphic mapping $z \rightarrow \operatorname{span}\left(\begin{array}{c}I \\ F(z)\end{array}\right) \in \operatorname{Gr}\left(n, \mathbf{C}^{2 n}\right)$; in view of the Calabi Rigidity Theorem, Theorem 1.2.2 above says the geometry of these realization operators mirrors the geometry of holomorphic curves in $\operatorname{Gr}\left(n, \mathrm{C}^{2 n}\right)$.

COROLlaRY 1.2.3. If there is a $z_{0}$ in D (unit disk) with $F\left(z_{0}\right)=G\left(z_{0}\right)=0$, then $S(g, F) \cong S(g, G) \Leftrightarrow$ there are constant unitary matrices $V, W$ such that $V F(z) W \equiv G(z)$ on $D$.

PROOF. From Theorems $\mathrm{A}, \mathrm{B}$ and 1.2 .2 , this corollary really says that $E_{F} \cong$ $E_{G} \Leftrightarrow \exists$ constant unitary matrices $V, W$ such that $V F(z) W \equiv G(z)$.

By the Calabi Rigidity Theorem, $E_{F} \cong E_{G} \Leftrightarrow \exists$ constant $2 n \times 2 n$ unitary matrix

$$
\mathcal{U}=\left(\begin{array}{ll}
U_{1} & U_{2} \\
U_{3} & U_{4}
\end{array}\right)
$$

where each $U_{j}$ is an $n \times n$ matrix, such that

$$
\left(\begin{array}{cc}
U_{1} & U_{2} \\
U_{3} & U_{4}
\end{array}\right)\left(\begin{array}{c}
I \\
F(z)
\end{array}\right) \equiv\left(\begin{array}{c}
I \\
G(z)
\end{array}\right) A(z)
$$

where $A(z)$ is an $n \times n$ invertible matrix for each $z \in D$.

" $\Rightarrow$ " We have the identity

$$
(I, 0) U^{*} U\left(\begin{array}{c}
I \\
F(z)
\end{array}\right)=A^{*}\left(z_{0}\right)(I, 0)\left(\begin{array}{c}
I \\
G(z)
\end{array}\right) A(z),
$$


if $z \in D$ which implies $I \equiv A^{*}\left(z_{0}\right) A(z)$ and therefore $A(z) \equiv A\left(z_{0}\right)$ is unitary.

But $U_{1}+U_{2} F(z) \equiv A\left(z_{0}\right)$ and $F\left(z_{0}\right)=0$ implies $A\left(z_{0}\right)=U_{1}$ is unitary, hence $U_{2}=U_{3}=0$ and $U_{4}$ is unitary.

So $U_{4} F(z) \equiv G(z) U_{1}$ in $D$.

" $\Leftarrow "\left(\begin{array}{cc}W^{*} & 0 \\ 0 & V\end{array}\right)\left(\begin{array}{c}I \\ F(z)\end{array}\right) \equiv\left(\begin{array}{c}I \\ G(z)\end{array}\right) W^{*}$ on $D$ gives $E_{F} \cong E_{G}$.

1.3. The first-order equivalence problem. We seek two operators $T_{1}, T_{2} \in$ $B_{n}(\Omega)$ such that $T_{1} \not \approx T_{2}$ but $\left.\left.T_{1}\right|_{\operatorname{ker}\left(T_{1}-w\right)^{2}} \cong T_{2}\right|_{\operatorname{ker}\left(T_{2}-w\right)^{2}}$ for each $w \in \Omega$.

Using Theorem $\mathrm{C}$ and Theorem 1.2.2, this problem is reduced to a geometric problem on $\operatorname{Gr}\left(n, \mathbf{C}^{2 n}\right)$, namely "Find two holomorphic curves $f_{1}, f_{2}$ in $\operatorname{Gr}\left(n, \mathbf{C}^{2 n}\right)$ such that $f_{1}^{*}\left(S\left(n, \mathbf{C}^{2 n}\right)\right)$ and $f_{2}^{*}\left(S\left(n, \mathbf{C}^{2 n}\right)\right)$ have the same curvature, but are inequivalent." Recall first that the Calabi Rigidity Theorem says $f_{1}^{*}\left(S\left(n, \mathbf{C}^{2 n}\right)\right) \cong$ $f_{2}^{*}\left(S\left(n, \mathbf{C}^{2 n}\right)\right) \Leftrightarrow f_{1}$ and $f_{2}$ are identical up to a unitary action of $\mathbf{C}^{2 n}$.

Second, fix an orthonormal basis of $\mathbf{C}^{2 n}$, say $e_{1}, \ldots, e_{2 n}$; then $\left(\left(e_{1}, \ldots, e_{2 n}\right) X\right.$, $\left.\left(e_{1}, \ldots, e_{2 n}\right) Y\right) \rightarrow Y^{\mathrm{T}} X$ is a nondegenerated bilinear form. It is not hard to see that it induces an automorphism of $\operatorname{Gr}\left(n, \mathbf{C}^{2 n}\right)$. Call this kind of automorphism a correlation of $\operatorname{Gr}\left(n, \mathbf{C}^{2 n}\right)$.

Recall the Plücker imbedding of $\operatorname{Gr}\left(n, \mathbf{C}^{2 n}\right) \rightarrow \mathbf{P}\left(\bigwedge^{n} \mathbf{C}^{2 n}\right)$ is the mapping $\operatorname{span}\left\{Z_{1}, \ldots, Z_{n}\right\} \rightarrow \operatorname{span}\left(Z_{1} \wedge Z_{2} \wedge \cdots \wedge Z_{n}\right)$.

If $\mathbf{P}\left(\bigwedge^{n} \mathbf{C}^{2 n}\right)$ carries the Fubini-Study metric, then the canonical Kähler structure of $\operatorname{Gr}\left(n, \mathbf{C}^{2 n}\right)$ is induced by this holomorphic imbedding. (See [Chern].)

With this metric on $\operatorname{Gr}\left(n, \mathbf{C}^{2 n}\right)$, every correlation of $\operatorname{Gr}\left(n, \mathbf{C}^{2 n}\right)$ is an isometry and is in fact the unique nontrivial isometric automorphism of $\operatorname{Gr}\left(n, \mathbf{C}^{2 n}\right)$ up to the action of $U(2 n)$ on $\operatorname{Gr}\left(n, \mathbf{C}^{2 n}\right)$. (See [Chow], [Cowen].)

Fix a correlation composed with a unitary $\left(\begin{array}{cc}0 & I \\ -I & 0\end{array}\right)$ action:

$$
\phi:\left(\begin{array}{c}
I \\
F
\end{array}\right) \rightarrow\left(\begin{array}{c}
-F^{\mathrm{T}} \\
I
\end{array}\right) \rightarrow\left(\begin{array}{c}
I \\
F^{\mathrm{T}}
\end{array}\right)=\left(\begin{array}{cc}
0 & I \\
-I & 0
\end{array}\right)\left(\begin{array}{c}
-F^{\mathrm{T}} \\
I
\end{array}\right),
$$

where $F$ is an $n \times n$ matrix, $I$ is the identity $n \times n$ matrix and $F^{\mathrm{T}}$ is the transpose of $F$. We shall show that for any holomorphic curve $f: \Omega \rightarrow \operatorname{Gr}\left(n, \mathbf{C}^{2 n}\right)$, $f^{*}\left(S\left(n, \mathbf{C}^{2 n}\right)\right)$ and $(\phi \circ f)^{*}\left(S\left(n, \mathbf{C}^{2 n}\right)\right)$ have the same curvature, but there is an $f$ such that we cannot get $\phi \circ f$ by any unitary action on $f$.

LEMMA 1.3.1. The vector bundles $E_{1}, E_{2}$ are equivalent to order one $\Leftrightarrow$ for any $C^{\infty}$ frame $S_{j}$ on $E_{j}(j=1,2), K_{1}\left(S_{1}\right)$ is similar to $K_{2}\left(S_{2}\right)$ pointwise.

PROOF. Notice that the matrix representation of curvature changes by similarity under change of frame and the curvature of the canonical connection is selfadjoint.

So $E_{1}$ is equivalent to order one with $E_{2} \Leftrightarrow$ the eigenvalues of $K_{1}$ and $K_{2}$ are the same.

In the following two lemmas, we write $F=\left(f_{i j}\right)_{i, j}, \tilde{F}=\left(\tilde{f}_{i j}\right)_{i, j}$, where all $f_{i j}$, $\tilde{f}_{i j}$ are analytic functions on $\Omega \subset \mathbf{C}$.

LEMMA 1.3.2. $E_{F}$ and $E_{F^{T}}$ are equivalent to order one.

PROOF. Over the holomorphic frame $\left[\begin{array}{c}I \\ F(z)\end{array}\right], K_{E}$ has matrix

$$
-\left(I+F^{*} F\right)^{-1}\left(F^{\prime}\right)^{*}\left(I+F F^{*}\right)^{-1} F^{\prime} .
$$


Using the elementary fact if $A, B$ are invertible matrices, then $A B \sim B A$ and $A \sim A^{\mathrm{T}}$, it is obvious that (**) is similar to

$$
\begin{aligned}
& -\left\{F^{\prime}\left(I+F^{*} F\right)^{-1}\left(F^{\prime}\right)^{*}\left(I+F F^{*}\right)\right\}^{\mathrm{T}} \\
& \quad=-\left(I+\left(F^{\mathrm{T}}\right)^{*} F^{\mathrm{T}}\right)^{-1}\left[\left(F^{\mathrm{T}}\right)^{\prime}\right]^{*}\left(I+F^{\mathrm{T}}\left(F^{\mathrm{T}}\right)^{*}\right)^{-1}\left(F^{\mathrm{T}}\right)^{\prime} .
\end{aligned}
$$

LEMMA 1.3.3. Fix $z_{0} \in \Omega$ and suppose that $F\left(z_{0}\right)=\tilde{F}\left(z_{0}\right)=0$, and

$$
F^{\prime}\left(z_{0}\right)=\tilde{F}^{\prime}\left(z_{0}\right)=\left(\begin{array}{ccc}
\lambda_{1} & & \\
& \ddots & \\
& & \lambda_{n}
\end{array}\right) \text { with }\left|\lambda_{i}\right| \neq\left|\lambda_{j}\right|(\text { if } i \neq j) .
$$

Then $E_{F} \cong E_{\tilde{F}}$ implies $\left|f_{i j}(z)\right| \equiv\left|\tilde{f}_{i j}(z)\right|$ for all $i, j$ and $z \in \Omega$.

PROOF. By Corollary 1.2.3,

$$
\begin{aligned}
& E_{F} \cong E_{\tilde{F}} \Leftrightarrow \exists \text { constant unitary } n \times n \text { matrices } \\
& V, W \text { such that } V F(z) W \cong \tilde{F}(z) \text { on } \Omega \\
& \Rightarrow V F^{\prime}\left(z_{0}\right) W=\tilde{F}^{\prime}\left(z_{0}\right) \\
& \Rightarrow W^{*}\left(\begin{array}{llll}
\left|\lambda_{1}\right|^{2} & & & \\
& \left|\lambda_{2}\right|^{2} & & \\
& & \ddots & \\
& & & \left|\lambda_{n}\right|^{2}
\end{array}\right) W \\
& =\left(\begin{array}{llll}
\left|\lambda_{1}\right|^{2} & & & \\
& \left|\lambda_{2}\right|^{2} & & \\
& & \ddots & \\
& & & \left|\lambda_{n}\right|^{2}
\end{array}\right) \\
& =V\left(\begin{array}{llll}
\left|\lambda_{1}\right|^{2} & & & \\
& \left|\lambda_{2}\right|^{2} & & \\
& & \ddots & \\
& & & \left|\lambda_{n}\right|^{2}
\end{array}\right) V^{*} \\
& \Rightarrow W, V \text { are both diagonal. }
\end{aligned}
$$

COROLlARY 1.3.4. Take any $F=\left(f_{i j}\right)_{i, j}$ with

1. $f_{i j} \in H^{\infty}$ and $f_{i j}(0)=0$ for all $i, j$;

2.

$$
F^{\prime}\left(z_{0}\right)=\left(\begin{array}{ccc}
\lambda_{1} & & \\
& \ddots & \\
& & \lambda_{n}
\end{array}\right), \quad\left|\lambda_{i}\right| \neq\left|\lambda_{j}\right|, \text { if } i \neq j ;
$$

3. $\left|f_{i j}\right| \neq\left|f_{j i}\right|$ for some $i, j$.

Then $E_{F} \not E_{F^{\mathrm{T}}}$.

We can now summarize the solution of the first-order equivalence problem as follows. 
THEOREM 1.3.5. If $n>1$, and $F$ is as in Corollary 1.3.4, then $S(z, F)$, $S\left(z, F^{\mathrm{T}}\right) \in B_{n}(D), S(z, F)$ and $S\left(z, F^{\mathrm{T}}\right)$ are equivalent to order one, but they are not unitarily equivalent.

1.4. The operator theoretical aspect of this realization. We begin with a powerful theorem of Brown-Douglas-Filmore [BDF].

THEOREM D [BDF]. Two essentially normal operators $T_{1}$ and $T_{2}$ are unitarily equivalent modulo compact operators $\Leftrightarrow \sigma_{e}\left(T_{1}\right)=\sigma_{e}\left(T_{2}\right)=X$ and $\operatorname{ind}\left(T_{1}-\lambda\right)=$ $\operatorname{ind}\left(T_{2}-\lambda\right)$ whenever $\lambda \in \mathbf{C}-X$.

Notice that similarity of the operators $T_{1}$ and $T_{2}$ already implies the conditions on the right of Theorem $\mathrm{D}$.

LEMMA 1.4.1. If $T, S$ are two bounded linear operators on $H$ such that $T$ is essentially normal and $[T, S]=0$, then

1. $\operatorname{Graph}(S)$ is invariant under $T \oplus T$;

2. $\left.T \oplus T\right|_{\mathrm{Graph}(S)}=T_{s}$ is unitarily equivalent to a compact perturbation of $T$.

Proof. 1 is trivial. For 2, note $T_{s} \sim T$ via the map $\phi: x \rightarrow(x, S x)$. In view of [BDF], it suffices to show $\left[T_{s}, T_{s}^{*}\right]$ is compact.

Let $P$ be the orthogonal projection of $H \oplus H$ onto $\operatorname{Graph}(S)$, then if $x \in H$,

$$
T_{s}^{*}(\phi(x))=P\left(T^{*} x \oplus T^{*} S x\right)=\phi\left(T^{*} x\right)+P\left(0 \oplus\left[T^{*}, S\right] x\right) .
$$

Define $K: \operatorname{Graph}(S) \rightarrow \operatorname{Graph}(S)$ by $K(\phi(x))=P\left(0 \oplus\left[T^{*}, S\right] x\right)$.

Note that by Fuglede's theorem in the Calkin algebra (i.e., $t s=s t, t^{*} t=t t^{*}$ " $\Rightarrow$ " $\left.t^{*} s=s t^{*}\right)\left[T^{*}, S\right]$ is compact, thus $K$ is a compact operator. Then

$$
T_{s}^{*}(\phi(x))=\phi\left(T^{*} x\right)+K(\phi(x))
$$

and

$$
\begin{aligned}
& T_{s} \circ T_{s}^{*}(\phi(x))=\phi\left(T \circ T^{*} x\right)+T_{s} \circ K(\phi(x)), \\
& T_{s}^{*} \circ T_{s}(\phi(x))=T_{s}^{*}(\phi(T x))=\phi\left(T^{*} \circ T x\right)+K \circ T_{s}(\phi(x)) .
\end{aligned}
$$

Thus $\left[T_{s}, T_{s}^{*}\right]=\phi \circ\left(\left[T, T^{*}\right]\right) \circ \phi^{-1}+\left[T_{s}, K\right]$.

THEOREM 1.4.2. If $g \in H^{\infty} \cap Q C$, then $S(g, F) \cong\left(T_{g \otimes I_{n}}^{*}+K\right) \sim T_{g \otimes I_{n}}^{*}$, where $K$ is a compact operator

$$
Q C=\overline{\left(H^{\infty}+C\left(S^{\prime}\right)\right)} \cap\left(H^{\infty}+C\left(S^{\prime}\right)\right) .
$$

ProOF. Since $g$ is quasi-continuous, $T_{g \otimes I_{n}}^{*}$ is essentially normal (see $[\mathbf{D}]$ ); the lemma above can then be applied.

We know very little about the compact operator $K$ in Theorem 1.4.2. One situation in which we do have some information is that of the following theorem, here stated without proof.

THEOREM 1.4.3. If $0<|a|<1$, then $S(z,(z-a) /(1-\bar{z} a)) \cong U_{+}^{*}+K$, where $U_{+}$is the unilateral shift on the orthonormal basis $\left\{e_{n}\right\}_{n=0}^{\infty}$ and $K\left(e_{j}\right)=0, j \geq 2$, $K\left(e_{0}\right)=\bar{a} e_{0}, K\left(e_{1}\right)=b e_{0}$ with $2|1+b|^{2}+|a|^{2}=1$. 


\section{PART 2. OPERATOR THEORETICAL REALIZATION OF TENSOR PRODUCT OF VECTOR BUNDLES}

We will use the definitions and notations introduced in Part 1. Besides, we shall use $\alpha=\left(\alpha^{(1)}, \alpha^{(2)}, \ldots, \alpha^{(n)}\right)$ to denote an ordered $n$-tuple of vectors in the Hilbert space $H$ (i.e. $\alpha^{(j)} \in H$ ), and define $\alpha^{*} \beta=\left(\left\langle\beta^{(j)}, \alpha^{(i)}\right\rangle\right)_{i, j}$, the $n \times n$ Gramian matrix of $\alpha$ and $\beta$, and $\|\alpha\|^{2} \stackrel{\text { def }}{=} \operatorname{tr}\left(\alpha^{*} \alpha\right)=\sum_{i=1}^{n}\left\|\alpha^{(i)}\right\|^{2}$. We shall say $\alpha \perp \beta$, if $\alpha^{*} \beta=0$.

Moreover, let $\alpha_{j}=\left(\alpha_{j}^{(1)}, \alpha_{j}^{(2)}, \ldots, \alpha_{j}^{(n)}\right)$ be a sequence of ordered $n$-tuple vectors, $j=1,2, \ldots$ We shall say $\left\{a_{j}\right\}_{j=1}^{\infty}$ is a linearly independent set, if $\left\{\alpha_{j}^{(k)}: 1 \leq k \leq\right.$ $n, j=1,2, \ldots\}$ is a linearly independent set in $H$. We write

$$
\operatorname{span}\left\{\alpha_{j}\right\}_{j=1}^{\infty} \stackrel{\text { def }}{=} \operatorname{span}\left\{\alpha_{j}^{(k)}: 1 \leq k \leq n, j=1,2, \ldots\right\} .
$$

Notice that if $\gamma_{1}$ is an ordered $m$-tuple vector and $\gamma_{2}$ is an ordered $n$-tuple vector, then $\gamma_{1} \otimes \gamma_{2}$ is an ordered $m n$-tuple vector.

2.1. The operator realization of tensor product of vector bundles. Theorem $\mathrm{A}$ in Part 1 says, for $T \in B_{n}(\Omega), T$ and $E_{T}$ are identified. Now, it is natural to ask: if $T_{1} \in B_{m}(\Omega)$ and $T_{2} \in B_{n}(\Omega)$, is $E_{T_{1}} \otimes E_{T_{2}}$ identified with some operator in $B_{m n}(\Omega)$ ?

The answer is affirmative, and hence we get a natural operation: $B_{m}(\Omega) \times$ $B_{n}(\Omega) \rightarrow B_{m n}(\Omega)$. We shall call this operation the "geometric tensor product."

DEFINITION 2.1.1. Let $T_{1} \in B_{m}(\Omega), T_{2} \in B_{n}(\Omega)$, where $T_{j}$ is defined on $H_{j}$ (separable Hilbert space), $j=1,2$. We define the subspace $H\left(T_{1}\right) * H\left(T_{2}\right)$ of $H_{1} \otimes H_{2}$ by

$$
H\left(T_{1}\right) * H\left(T_{2}\right)=\underset{z \in \Omega}{\operatorname{span}}\left[\operatorname{ker}\left(T_{1}-z\right) \otimes \operatorname{ker}\left(T_{2}-z\right)\right]
$$

and the operator $T_{1} * T_{2}$ by

$$
\left.T_{1} * T_{2} \stackrel{\text { def }}{=}\left(T_{1} \otimes I\right)\right|_{H\left(T_{1}\right) * H\left(T_{2}\right)} .
$$

Observe that $H\left(T_{1}\right) * H\left(T_{2}\right)$ is a common invariant subspace of $T_{1} \otimes I$ and $I \otimes T_{2}$. Thus $T_{1} * T_{2}$ is well defined. Moreover $\left.\left(T_{1} \otimes I\right)\right|_{H\left(T_{1}\right) * H\left(T_{2}\right)}=\left.\left(I \otimes T_{2}\right)\right|_{H\left(T_{1}\right) * H\left(T_{2}\right)}$ and $\left\|T_{1} * T_{2}\right\| \leq \min \left(\left\|T_{1}\right\|,\left\|T_{2}\right\|\right)$.

LEMMA 2.1.1. Let $W$ be any open subset of $\Omega$, then

$$
H\left(T_{1}\right) * H\left(T_{2}\right)=\operatorname{span}_{z \in W}\left[\operatorname{ker}\left(T_{1}-z\right) \otimes \operatorname{ker}\left(T_{2}-z\right)\right]
$$

where $T_{1}, T_{2}$ are as in Definition 2.1.1.

PrOOF. Let $\gamma_{j}$ be a global holomorphic frame of $E_{T_{j}}$ (cf. $\left.[\mathbf{G}]\right), j=1,2$. By Definition 2.1.1,

$$
H\left(T_{1}\right) * H\left(T_{2}\right)=\operatorname{span}\left\{\gamma_{1}(z) \otimes \gamma_{2}(z): z \in \Omega\right\}
$$

Also,

$$
\operatorname{span}_{z \in W}\left[\operatorname{ker}\left(T_{1}-z\right) \otimes \operatorname{ker}\left(T_{2}-z\right)\right]=\operatorname{span}\left\{\gamma_{1}(z) \otimes \gamma_{2}(z): z \in W\right\}
$$

The lemma then follows from the application of the Identity Theorem in complex analysis. 
THEOREM 2.1.2. Let $T_{1} \in B_{m}(\Omega)$ and $T_{2} \in B_{n}(\Omega)$, then $T_{1} * T_{2} \in B_{m n}(\Omega)$ and $E_{T_{1} * T_{2}}=E_{T_{1}} \otimes E_{T_{2}}$.

Proof. Step 1. We claim that for each $z \in \Omega$,

$$
\operatorname{ker}\left(T_{1} * T_{2}-z\right)=\operatorname{ker}\left(T_{1}-z\right) \otimes \operatorname{ker}\left(T_{2}-z\right) .
$$

Fix $z \in \Omega$, since $\left(T_{1} * T_{2}\right)-z=\left.\left[\left(T_{1}-z\right) \otimes I\right]\right|_{H\left(T_{1}\right) * H\left(T_{2}\right)}$, clearly $\operatorname{ker}\left[\left(T_{1} * T_{2}\right)-z\right] \supset$ $\operatorname{ker}\left(T_{1}-z\right) \otimes \operatorname{ker}\left(T_{2}-z\right)$.

Conversely, for any $x \in H_{1} \otimes H_{2}$ write $x=x_{1}+x_{2}+y$, where

$x_{1} \in \operatorname{ker}\left(T_{1}-z\right) \otimes \operatorname{ker}\left(T_{2}-z\right), \quad x_{2} \in \operatorname{ker}\left(T_{1}-z\right) \otimes\left[\operatorname{ker}\left(T_{2}-z\right)\right]^{\perp}$,

$$
y \in\left[\operatorname{ker}\left(T_{1}-z\right)\right]^{\perp} \otimes H_{2} .
$$

Notice that since $\left(T_{1}-z\right) \otimes I$ and $I \otimes\left(T_{2}-z\right)$ are both onto, as linear mappings $\left.\left[\left(T_{1}-z\right) \otimes I\right]\right|_{\left[\operatorname{ker}\left(T_{1}-z\right)\right]^{\perp} \otimes H_{2}}$ and $\left.\left[I \otimes\left(T_{2}-z\right)\right]\right|_{H_{1} \otimes\left[\operatorname{ker}\left(T_{2}-z\right)\right]^{\perp}}$ are both invertible.

Now, if $x \in \operatorname{ker}\left(T_{1} * T_{2}-z\right)$, then $\left[\left(T_{1}-z\right) \otimes I\right] y=\left[\left(T_{1} * T_{2}\right)-z\right] x=0$, so $y=0$; moreover $\left[I \otimes\left(T_{2}-z\right)\right] x_{2}=\left[\left(T_{1} * T_{2}\right)-z\right] x=0$, so $x_{2}=0$. Thus $x=x_{1} \in \operatorname{ker}\left(T_{1}-z\right) \otimes \operatorname{ker}\left(T_{2}-z\right)$.

Step 2. We claim $T_{1} * T_{2}-z$ is onto for each $z \in \Omega$. (Notice that $T_{1} * T_{2} \in B_{m n}(\Omega)$ will then follow from Step 1 and Step 2.)

Since $T_{1} * T_{2}-z=\left(T_{1}-z\right) *\left(T_{2}-z\right)$ (by Definition 2.1.1), it will be enough if we assume $0 \in \Omega$ and get a right inverse of $T_{1} * T_{2}$.

Let $S_{j}=T_{j}^{*}\left(T_{j} T_{j}^{*}\right)^{-1}, j=1,2$. It is easy to see (1) $T_{j} S_{j}=$ id for $j=1,2$; (2) if $e_{j}$ is an orthonormal basis of $\operatorname{ker} T_{j}(j=1,2)$, then $\left(1-z S_{j}\right)^{-1} e_{j}$ is a local holomorphic frame of $E_{T_{j}}$ near $0(j=1,2)$ and $e_{j} \perp S_{j}^{k} e_{j}$ for all $k \geq 1$.

Let $[|z|<r] \subset \Omega$ such that the two series $\sum_{k=0}^{\infty} z^{k}\left(S_{j}^{k} e_{j}\right), j=1,2$, both converge in this disk. Then by Lemma 2.1.1,

$$
\underset{0<|z|<r}{\operatorname{span}}\left\{\left[\sum_{k=0}^{\infty} z^{k}\left(S_{1}^{k} e_{1}\right)\right] \otimes\left[\sum_{k=0}^{\infty} z^{k}\left(S_{2}^{k} e_{2}\right)\right]\right\}=H\left(T_{1}\right) * H\left(T_{2}\right) .
$$

If $P$ is the orthogonal projection of $H_{1}$ onto $\operatorname{ker} T_{1}$, then the following computation shows $H\left(T_{1}\right) * H\left(T_{2}\right)$ is invariant under $\left(S_{1} \otimes I\right)+\left(P \otimes S_{2}\right)$ :

$$
\begin{aligned}
{\left[\left(S_{1} \otimes I\right)+\left(P \otimes S_{2}\right)\right]\left\{\left(\sum_{k=0}^{\infty} z^{k} S_{1}^{k} e_{1}\right) \otimes\left(\sum_{k=0}^{\infty} z^{k} S_{2}^{k} e_{2}\right)\right\} } \\
\quad=\left[\sum_{k=0}^{\infty} z^{k} S_{1}^{k+1} e_{1}\right] \otimes\left[\sum_{k=0}^{\infty} z^{k} S_{2}^{k} e_{2}\right]+e_{1} \otimes\left[\sum_{k=0}^{\infty} z^{k} S_{2}^{k+1} e_{2}\right] \\
\quad=\frac{1}{z}\left\{\left[\sum_{k=1}^{\infty} z^{k} S_{1}^{k} e_{1}\right] \otimes\left[\sum_{k=0}^{\infty} z^{k} S_{2}^{k} e_{2}\right]\right\}+\frac{1}{z}\left\{e_{1} \otimes\left[\sum_{k=1}^{\infty} z^{k} S_{2}^{k} e_{2}\right]\right\} \\
\quad=\frac{1}{z}\left\{\left[\sum_{k=0}^{\infty} z^{k} S_{1}^{k} e_{1}\right] \otimes\left[\sum_{k=0}^{\infty} z^{k} S_{2}^{k} e_{2}\right]\right\}-\frac{1}{z}\left[e_{1} \otimes e_{2}\right],
\end{aligned}
$$

for all $0<|z|<r$.

Now, it becomes clear that $\left(S_{1} \otimes I\right)+\left(P \otimes S_{2}\right)$ is a right inverse of $T_{1} * T_{2}$.

Finally, $T_{1} * T_{2}$ is identified with $E_{T_{1}} \otimes E_{T_{2}}$, i.e. $E_{T_{1} * T_{2}}=E_{T_{1}} \otimes E_{T_{2}}$ is a trivial consequence of Step 1. 
2.2. Partial transformation induced by geometric tensor product. Fix $S \in B_{1}\left(\Omega_{1}\right)$ and consider the transformation

$$
T \rightarrow S * T
$$

$\left(B_{n}\left(\Omega_{2}\right)\right.$ to $\left.B_{n}\left(\Omega_{1} \cap \Omega_{2}\right)\right)$. We shall prove that if $S$ is almost the backward unilateral shift then $S * T \sim S \otimes$ id $_{\mathbf{C}^{n}}$.

The philosophy of this proof is that "we can $\operatorname{read} T \in B_{n}(\Omega)$ in a different way by reading $E_{T}$ in a different way."

DEFINITION 2.2.1. Let $T \in B_{n}(\Omega)$, where $\Omega$ is a component of $\mathbf{C}-\sigma_{e}(T)$. A holomorphic frame $\gamma$ of $E_{T}$ (defined near $z_{0} \in \Omega$ ) will be said to be normalized at $z_{0}$ if $\gamma^{*}\left(z_{0}\right) \gamma(z) \equiv I_{n}$ wherever $\gamma$ is defined.

Notice that if $\gamma$ is as in the definition above, then $\gamma\left(z_{0}\right) \perp\left(\partial^{k} \gamma / \partial z^{k}\right)\left(z_{0}\right)$ for all $k \geq 1$.

DEFINITION 2.2.2. If $T \in B_{n}(\Omega)$ and $\Omega$ is a component of $\mathbf{C}-\sigma_{e}(T)$, then for each $z_{0} \in \Omega$, we define the number $R\left(T, z_{0}\right)$ by

$$
\begin{aligned}
\max \left\{r \leq d\left(z_{0}, \sigma_{e}(T)\right):\right. & \text { for each }\left|z-z_{0}\right|<r, \text { there is no nontrivial } \\
& \text { subspace of } \left.\operatorname{ker}(T-z) \text { perpendicular to } \operatorname{ker}\left(T-z_{0}\right)\right\} .
\end{aligned}
$$

(Notice: there is no nontrivial subspace of $\operatorname{ker}(T-z)$ perpendicular to $\operatorname{ker}\left(T-z_{0}\right)$ $\Leftrightarrow$ for any basis $\gamma_{z}$ of $\operatorname{ker}(T-z)$ and basis $\gamma_{z_{0}}$ of $\operatorname{ker}\left(T-z_{0}\right)$, we have $\operatorname{det}\left[\gamma_{z_{0}}^{*} \gamma_{z}\right] \neq 0$.)

It is easy to see that $R\left(T, z_{0}\right)$ is invariant under unitary equivalence of $T$ and $R\left(T, z_{0}\right)>0$ always.

LEMMA 2.2.1. Let $T \in B_{n}(\Omega)$ and assume $\Omega$ is a component of $\mathbf{C}-\sigma_{e}(T)$. Fix $z_{0} \in \Omega$. Then there is a holomorphic frame defined on the entire disk $\left\{z:\left|z-z_{0}\right|<\right.$ $\left.R\left(T, z_{0}\right)\right\}$ which is normalized at $z_{0}$.

PrOOF. Let $\tilde{\gamma}$ be a global holomorphic frame of $E_{T}$ on $\Omega$; then

$$
\gamma(z)=\tilde{\gamma}(z)\left[\tilde{\gamma}^{*}\left(z_{0}\right) \tilde{\gamma}(z)\right]^{-1}\left[\tilde{\gamma}^{*}\left(z_{0}\right) \tilde{\gamma}\left(z_{0}\right)\right]^{1 / 2}
$$

works.

LEMMA 2.2.2. Let $0 \in \Omega, T \in B_{n}(\Omega)$ and $0<r<R(T, 0)$. Let $\gamma$ be the holomorphic frame of $E_{T}$ as defined in the lemma above. Then there is an orthonormal basis $\left\{e_{j}^{(k)}: j \geq 0,1 \leq k \leq n\right\}\left(e_{j} \stackrel{\text { def }}{=}\left(e_{j}^{(1)}, e_{j}^{(2)}, \ldots, e_{j}^{(n)}\right)\right)$ such that $\gamma(z)=e_{0}+\sum_{j=1}^{\infty} e_{j} B_{j}(z)$, where the $B_{j}(z)$ 's are $n \times n$ matrices of analytic functions on $|z|<R(T, 0)$. Moreover, if $\left\|B_{j}\right\|_{r}^{2} \stackrel{\text { def }}{=} \sup _{|z| \leq r}\left[\operatorname{Tr}\left(B_{j}^{*}(z) B_{j}(z)\right)\right]$, then $\sum_{j=1}^{\infty}\left\|B_{j}\right\|_{r}^{2}<+\infty$.

ProOF. Notice that $\left\{\left(\partial^{k} \gamma / \partial z^{k}\right)(0)\right\}_{k=0}^{\infty}$ is a linearly independent set spanning $H$ (see $[\mathbf{C}-\mathbf{D}, \mathbf{1}, \S 1])$. Also $\gamma(0) \perp\left(\partial^{k} \gamma / \partial z^{k}\right)(0)$ for all $k \geq 1$ and $\gamma(0)$ is an orthonormal set.

We shall show that Gram-Schmidt orthonormalization $\left\{e_{j}\right\}_{j=0}^{\infty}$ of the set $\left\{\left(\partial^{k} \gamma / \partial z^{k}\right)(0)\right\}_{k=0}^{\infty}$ is a right choice of our orthonormal basis.

Let $H_{j}=\operatorname{span}\left\{\left(\partial^{k} \gamma / \partial z^{k}\right)(0): 0 \leq k \leq j\right\}$ for each $j \geq 0$.

Let $e_{0}=\gamma(0)$ and, for each $j \geq 1$, let $e_{j}=\left(e_{j}^{(1)}, \ldots, e_{j}^{(n)}\right)$ be an orthonormal basis of $H_{j} \ominus H_{j-1}$. 
Thus $\left\{e_{j}^{(l)}: 1 \leq l \leq n, j=0,1,2, \ldots\right\}$ is an orthonormal basis of $H$ and for each $k \geq 1$ we have

$$
\frac{1}{k !}\left(\frac{\partial^{k}}{\partial z^{k}} \gamma\right)(0)=\sum_{i=1}^{k} e_{i} A_{i k}
$$

where $A_{i k}$ is an $n \times n$ constant matrix.

Now $\gamma(z)=\sum_{j=1}^{\infty}\left(\sum_{i=1}^{j} e_{i} A_{i j}\right) z^{j}+e_{0}$ on $|z|<R(T, 0)$ and $\sum_{j=1}^{\infty} A_{i j} z^{j}=e_{i}^{*} \gamma(z)$ is convergent in $M(n, \mathrm{C})$.

Let $\sum_{j=1}^{\infty} A_{i j} z^{j}=z^{i} \tilde{A}_{i}(z)=B_{i}(z)$. Then

$$
\gamma(z)=e_{0}+\sum_{i=1}^{\infty} e_{i} B_{i}(z) \text { for }|z|<R(T, 0) .
$$

For $A \in M(n, \mathbf{C})$, write $\|A\|^{2}=\operatorname{tr}\left(A^{*} A\right)$.

Assume $0<r<\delta<R(T, 0)$ and $\|\gamma(z)\|=\sum_{j=0}^{\infty}\left\|z^{i} \tilde{A}_{i}(z)\right\|^{2} \leq M$ for $|z| \leq \delta$.

By the maximum principle, on $|z| \leq \delta$, we have

$$
\left\|\delta^{i} \tilde{A}_{i}(z)\right\|^{2} \leq \sup _{|\varsigma|=\delta}\left\|\varsigma^{i} \tilde{A}_{i}(\varsigma)\right\|^{2} \leq \sup _{|\varsigma|=\delta}\|\gamma(\varsigma)\|^{2} \leq M
$$

Thus

$$
\begin{aligned}
\sum_{i=1}^{\infty}\left\|B_{i}\right\|_{r}^{2} & =\sum_{i=1}^{\infty} \sup _{|z| \leq r}\left\|z^{i} \tilde{A}_{i}(z)\right\|^{2} \leq \sum_{i=1}^{\infty} \sup _{|z| \leq r}\left\|\zeta^{i} \tilde{A}_{i}(z)\right\|^{2}\left(\frac{r}{\delta}\right)^{2 i} \\
& \leq M \sum_{i=1}^{\infty}\left(\frac{r}{\delta}\right)^{2 i}<+\infty .
\end{aligned}
$$

Our next lemma is a generalization of Lemma 1.1.1.

If $A \in H^{\infty} \otimes M(n, \mathbf{C})$, let $\|A\|_{\infty}^{2} \stackrel{\text { def }}{=}\left\|\operatorname{tr} A^{*} A\right\|_{\infty}$. It is easy to see $\left\|T_{A}^{*}\right\| \leq\|A\|_{\infty}$, where $T_{A}$ is the matrix analytic Toeplitz operator acting on $H^{2} \otimes \mathbf{C}^{n}=\tilde{H}$ (as row vectors).

Now, if $\left\{F_{k}\right\}_{k=1}^{\infty}$ is a sequence of $n \times n$ matrices, each of them having all entries in $H^{\infty}$, with $\sum_{k=1}^{\infty}\left\|F_{k}\right\|_{\infty}^{2}<\infty$, then $\phi: \tilde{H} \rightarrow \tilde{H} \oplus \tilde{H} \oplus \cdots$ defined by

$$
f \rightarrow f \oplus T_{F_{1}}^{*} f \oplus T_{F_{2}}^{*} f \oplus \cdots
$$

is clearly a bounded linear and bounded below mapping; denote its range by $R$.

LEMMA 2.2.3. If $g \in H^{\infty} \cap Q C$, let $S_{R}(g)$ denote $\left.\left(T_{g \otimes I_{n}}^{*} \oplus T_{g \otimes I_{n}}^{*} \oplus \cdots\right)\right|_{R}$. Then $S_{R}(g) \cong\left(T_{g \otimes I_{n}}^{*}+K\right) \sim T_{g \otimes I_{n}}^{*}$, where $K$ is a compact operator.

PROOF. The operator $S_{R}(g)$ is similar to $T_{g \otimes I_{n}}^{*}$ via $\phi$. By [BDF], it suffices to show $S_{R}(g)$ is essentially normal. Let $P$ be the orthogonal projection of $\tilde{H} \oplus \tilde{H} \oplus \cdots$ onto $R$, then

$$
\begin{aligned}
\left(S_{R}(g)\right)^{*}(\phi(f)) & =P\left\{T_{g \otimes I_{n}}(f) \oplus\left(T_{g \otimes I_{n}} \circ T_{F_{1}}^{*}(f)\right) \oplus\left(T_{g \otimes I_{n}}^{*} \circ T_{F_{2}}^{*}(f)\right) \oplus \cdots\right\} \\
& =\phi\left(T_{g \otimes I_{n}}(f)\right)+P\left(0 \oplus L_{1}(f) \oplus L_{2}(f) \oplus \cdots\right)
\end{aligned}
$$

where $L_{j}=\left[T_{g \otimes I_{n}}, T_{F_{j}}^{*}\right]$ which is compact.

Notice that $\left\|L_{j}\right\| \leq 2\|g\|_{\infty} \circ\left\|F_{j}\right\|_{\infty}, j \geq 1$. 
Denote the bounded linear mapping $f \rightarrow 0 \oplus L_{1}(f) \oplus L_{2}(f) \oplus \cdots$ by $0 \oplus L_{1} \oplus$ $L_{2} \oplus \cdots(\tilde{H}$ into $\tilde{H} \oplus \tilde{H} \oplus \cdots)$.

Set $K_{\infty}=P \circ\left(0 \oplus L_{1} \oplus L_{2} \oplus \cdots\right) \circ \phi^{-1}$ and set

$$
K_{j}=P \circ\left(0 \oplus L_{1} \oplus \cdots \oplus L_{j-1} \oplus L_{j} \oplus 0 \oplus 0 \oplus \cdots\right) \circ \phi^{-1} .
$$

Then $K_{j}$ is compact and $K_{j} \rightarrow K_{\infty}$ follows from

$$
\left\|\left(K_{\infty}-K_{j}\right) \phi(f)\right\|^{2} \leq \sum_{k=j+1}^{\infty}\left\|L_{k}(f)\right\|_{\infty}^{2} \leq\left\{4\|g\|_{\infty}^{2}\left(\sum_{k=j+1}^{\infty}\left\|F_{k}\right\|_{\infty}^{2}\right)\right\} \circ\|f\|^{2} .
$$

Therefore $K_{\infty}$ is compact.

Finally, using $\left(S_{R}(g)\right)^{*}(\phi(f))=\phi\left(T_{g \otimes I_{n}}(f)\right)+K_{\infty}(\phi(f))$, we can directly check

$$
\left[S_{R}(g),\left(S_{R}(g)\right)^{*}\right]=\phi \circ\left[T_{g \otimes I_{n}}^{*}, T_{g \otimes I_{n}}\right] \circ \phi^{-1}+\left[S_{R}(g), K_{\infty}\right] .
$$

Now, we are ready to prove the main result of this section.

THEOREM 2.2.4. For any $T \in B_{n}(\Omega)$ (where $\Omega$ is a component of $\mathbf{C}-\sigma_{e}(T)$ ) and $z_{0} \in \Omega$, let $0<r<R\left(T, z_{0}\right)$ and $g(z)=z_{0}+r z$. Then

$$
T_{g}^{*} * T \cong\left(T_{g \otimes I_{n}}^{*}+K\right) \sim T_{g \otimes I_{n}}^{*},
$$

where $K$ is a compact operator.

PROOF. Without loss of generality, we assume $z_{0}=0 \in \Omega$. Let $\gamma(z),\left\{B_{j}(z)\right\}_{j=1}^{\infty}$ be as in Lemma 2.2.2, and write $B_{j}(z)=\overline{B_{j}(\bar{z})}$. Then by Lemma 2.2.2, the linear mapping

$$
\phi: f \rightarrow f \oplus T_{B_{1} \circ g}^{*}(f) \oplus T_{B_{2} \circ g}^{*}(f) \oplus \cdots
$$

is bounded and clearly bounded below. Let $R_{T}=\operatorname{range}(\phi)$ and

$$
S_{R_{T}}(g)=\left.\left(T_{g \otimes I_{n}}^{*} \oplus T_{g \otimes I_{n}}^{*} \oplus \cdots\right)\right|_{R_{T}} .
$$

By Lemma 2.2.3, the theorem will be true once we show $S_{R_{T}}(g) \cong T_{g}^{*} * T$. This last equivalence is achieved by the holomorphic isometric bundle map from $E_{S_{R_{T}}}(g)$ to $E_{T_{g}^{* * T}}$ (both over the disk $r D$ )

$$
\left[k_{\mathcal{G}(z)} \otimes I_{n}\right] \oplus B_{1}(z) k_{\mathcal{G}(z)} \oplus B_{2}(z) k_{\mathcal{G}(z)} \oplus \cdots \rightarrow k_{\mathcal{G}(z)} \otimes \gamma(z)
$$

where $\mathcal{G}(z)=\overline{g^{-1}(\bar{z})}$ and $k_{z}(\xi)=1 /(1-\xi z)$ is the reproducing kernel of the Hardy space.

COROLLARY 2.2.5. If $z_{0}=0$ and $T, \Omega, g, r$ are all as above, then $\left\|T_{g}^{*} * T\right\|=$ $\left\|T_{g}^{*}\right\|=r$.

Proof. By Definition 2.2.1, $\left\|T_{g}^{*} * T\right\| \leq\left\|T^{*}\right\|=r$; also by Weyl's theorem about the spectrum of a compact perturbation (see $[\mathbf{H}]$ ),

$$
\left\|T_{g \otimes I_{n}}^{*}+K\right\|=r\left\|T_{z \otimes I_{n}}+K / r\right\| \geq r .
$$

COROLLARY 2.2.6. If $z_{0}=0$ and $T \in B_{n}(\Omega), R(T, 0)>1$, then

$$
T_{z}^{*} * T \cong\left(T_{z \otimes I_{n}}^{*}+K\right) \sim T_{z \otimes I_{n}}^{*},
$$

where $K$ is a compact operator. 
DEFINITION 2.2.3. Let $J$ be the set of Cowen-Douglas operators $T$ with $0 \in$ C- $\sigma_{e}(T)$ and $R(T, 0)>1$. Then we define the transformation $\psi: J \rightarrow \bigcup_{n=1}^{\infty} B_{n}(D)$ by $\psi(T)=T_{z}^{*} * T$.

What is this transformation good for? In addition to Corollaries 2.2.5, 2.2.6, we have " $T_{1}$ and $T_{2}$ are equivalent to order $k \Leftrightarrow \psi\left(T_{1}\right)$ and $\psi\left(T_{2}\right)$ are equivalent to order $k$ (in particular, $T_{1} \cong T_{2} \Leftrightarrow \psi\left(T_{1}\right) \cong \psi\left(T_{2}\right)$ )," " $T$ and $\psi(T)$ have the same reducibility." Some other properties of this transformation were discussed in [L].

Before we studied the transformation, we had the Fourier and Laplace transformations in mind. But it turns out the flavor is quite different. Further modification of the transformation or a new way of studying this transformation is expected.

\subsection{The square transformation induced by geometric tensor product.}

DEFINITION 2.3.1. We define the square transformation $\Gamma: B_{1}(\Omega) \rightarrow B_{1}(\Omega)$ by $\Gamma(T)=T * T$.

THEOREM 2.3.1. If $T_{1}, T_{2} \in B_{1}(\Omega)$ and $T_{1} \sim T_{2}$, then $\Gamma\left(T_{1}\right) \sim \Gamma\left(T_{2}\right)$.

ProOF. Let $T_{2}=Q^{-1} T_{1} Q$, where $Q$ is an invertible operator. Then $T_{2} \otimes I=$ $\left(Q^{-1} \otimes Q^{-1}\right)\left(T_{1} \otimes I\right)(Q \otimes Q)$.

Notice that

$$
H\left(T_{2}\right) * H\left(T_{2}\right)=\left(Q^{-1} \otimes Q^{-1}\right)\left[H\left(T_{1}\right) * H\left(T_{1}\right)\right] .
$$

Now it is easy to check that the following diagram commutes:

$$
\begin{array}{ccc}
H\left(T_{2}\right) * H\left(T_{2}\right) \stackrel{T_{2} \otimes I}{\longrightarrow} & H\left(T_{2}\right) * H\left(T_{2}\right) \\
Q^{-1} \otimes Q^{-1} \uparrow & Q^{-1} \otimes Q^{-1} \uparrow \\
H\left(T_{1}\right) * H\left(T_{1}\right) \stackrel{T_{1} \otimes I}{\longrightarrow} H\left(T_{1}\right) * H\left(T_{1}\right) .
\end{array}
$$

Since the curvature of $T$ is exactly one half of the curvature of $\Gamma(T)$, so we have

THEOREM 2.3.2. If $T_{1}, T_{2} \in B_{1}(\Omega)$, then $T_{1} \cong T_{2} \Leftrightarrow \Gamma\left(T_{1}\right) \cong \Gamma\left(T_{2}\right)$.

THEOREM 2.3.3. If $g \in H^{\infty}$ and $T_{g}^{*} \in B_{1}(\Omega)$, then $\Gamma\left(T_{g}^{*}\right) \cong B_{g}^{*}$, the corresponding Toeplitz operator on the Bergman space.

Proof. From Lemma 1.2.1, we know $g: g^{-1}(\bar{\Omega}) \rightarrow \bar{\Omega}$ is a conformal equivalence. For $B_{g}^{*}$, if we repeat the proof of Lemma 1.2 .1 word by word, it is easy to see $B_{g}^{*} \in B_{1}(\Omega)$.

Let $\beta_{z}(\xi)=\pi^{-1}(1-\xi z)^{-2}$ be the Bergman kernel. Recall $B_{g}^{*}\left(\beta_{z}\right)=\overline{g(\bar{z})} \beta_{z}$, so the mapping

$$
k_{\overline{g^{-1}(\bar{z})}} \otimes k_{\overline{g^{-1}(\bar{z})}} \rightarrow \beta_{\overline{g^{-1}(\bar{z})}} \quad(z \in \Omega)
$$

gives a holomorphic isometric bundle map from $E_{T_{g}^{*} T_{g}^{*}}$ to $E_{B_{g}^{*}}$.

It is well known that for each $\psi \in C(\cos (D)), T_{\phi_{S_{S} 1}}$ and $B_{\phi}$ are unitarily equivalent up to compact perturbation (see $[\mathbf{C O}]$ ). We pose a problem here (related to Theorem 2.3.3):

Let $\psi \in C\left(S^{1}\right)$ with harmonic extension $\hat{\phi}$ to $D$. If $T_{\phi} \in B_{1}(\Omega)$, do we have $\Gamma\left(T_{\phi}\right) \cong B_{\hat{\phi}}$ ?

We hope this approach can lead to a rediscovery of the fact: $B_{z}^{*}$ is not unitarily equivalent to any Toeplitz operator on Hardy space. 


\section{REFERENCES}

[BDF] L. G. Brown, R. G. Douglas, and P. A. Filmore, Unitary equivalence modulo the compact operators and extensions of $C^{*}$-algebras, Proc. Conf. on Operator Theory, Lecture Notes in Math., vol. 345, Springer-Verlag, 1973, pp. 58-128.

[C-D,1] M. J. Cowen and R. G. Douglas, Complex geometry and operator theory, Acta Math. 141 (1978), 187-261.

[C-D,2] _ Equivalence of connections, Adv. in Math. 56 (1985), 39-91.

[CO] L. A. Coburn, Singular integral operators and Toeplitz operators on odd spheres, Indiana Univ. Math. J. 23 (1973), 433-439.

[Chern] Shing-shen Chern, Complex manifolds without potential theory, Springer-Verlag, 1979.

[Chow] Wei-Liang Chow, On the geometry of algebraic homogeneous spaces, Ann. of Math. (2) 50 (1949), 32-67.

[Cowen] M. J. Cowen, Automorphisms of Grassmannians, ubpublished.

[D] R. G. Douglas, Banach algebra techniques in operator theory, Pure and Appl. Math., Vol. 49, Academic Press, New York, 1972.

[G] H. Grauert, Analytische Faserungen über holomorphvollständigen Räumen, Math. Ann. 135 (1958), 263-273.

[H] P. R. Halmos, A Hilbert space problem book, Springer-Verlag, New York, 1982.

[L] Lin Qing, Imbedded operators and geometric tensor product, Ph.D. Dissertation, SUNY, Buffalo, N. Y., 1986.

Department of Mathematics, University of Kansas, LaWRENCE, Kansas 66045

Current address: Institute of Mathematics, Academia Sinica, Beijing, China 